\title{
Assessment of heavy rainfall-induced disaster potential based on an ensemble simulation of Typhoon Talas (2011) with controlled track and intensity
}

\author{
Y. Oku ${ }^{1, *}$, J. Yoshino ${ }^{2}$, T. Takemi ${ }^{3}$, and H. Ishikawa ${ }^{3}$ \\ ${ }^{1}$ Osaka City Institute of Public Health and Environmental Sciences, Osaka, Japan \\ ${ }^{2}$ Graduate School of Engineering, Gifu University, Gifu, Japan \\ ${ }^{3}$ Disaster Prevention Research Institute, Kyoto University, Kyoto, Japan \\ *now at: School of Human Science and Environment, University of Hyogo, Himeji, Japan
}

Correspondence to: Y. Oku (oku@storm.dpri.kyoto-u.ac.jp)

Received: 14 May 2014 - Published in Nat. Hazards Earth Syst. Sci. Discuss.: 19 June 2014

Revised: - - Accepted: 25 August 2014 - Published: 7 October 2014

\begin{abstract}
Typhoon Talas (2011) caused extensive damage through landslides on the Kii Peninsula, Japan, in September 2011. The purpose of the present study is to quantitatively describe the potential for the occurrence of heavy rainfallinduced disasters if the typhoon track perturbs slightly or the typhoon intensifies. Regarding to the consideration of the track displacement of the typhoon, a procedure is proposed to generate different typhoon tracks perturbed from the original track of the typhoon. In this procedure, the position of a typhoon is artificially shifted at a certain time before landing in a physically consistent manner by applying potential vorticity inversion (PVI) methodology. After relocating the typhoon, the subsequent progress is simulated by a mesoscale meteorological model. Using the output, which consists of a set of realizations having different typhoon tracks, the worst-case scenario is discussed in terms of the soil water index (SWI) of the Kii Peninsula. The SWI is an indicator of the amount of water in soil that represents the hazard of landslide disasters. The maximum spatially averaged SWI is 1.10 times as large as that from the original typhoon track. Regarding the consideration of severer typhoon, the same method is used, but the intensity of the potential vorticity of a typhoon is artificially modified at the position instead of relocating potential vorticity to a different place. The maximum spatially averaged SWI is 1.28 times as large as that of the original typhoon intensity.
\end{abstract}

\section{Introduction}

Tropical cyclones (TCs) and their relationship to global climate change have been the subject of considerable attention among scientists in meteorology and disaster science. From the viewpoint of disaster mitigation or adaptation, the media and political advocates have paid particular attention to a possible effect of human-caused climate change in patterns of TC activity, such as frequency, intensity and track of TCs.

The Special Report on Managing the Risks of Extreme Events and Disasters to Advance Climate Change Adaptation (SREX, IPCC, 2012) reported that "the average TC maximum wind speed is likely to increase, although increases may not occur in all ocean basins. The global frequency of TCs will likely either decrease or remain essentially unchanged. However, heavy rainfall associated with TCs is also likely to increase with continued global warming." On the other hand, the impact of an individual TC can vary greatly depending on when and where it makes landfall, in addition to its strength.

In September 2011, Typhoon Talas (2011) brought heavy rainfall to Japan, and the Kii Peninsula especially experienced record-breaking rainfall. The typhoon reached its peak intensity with a maximum sustained wind speed of $25.7 \mathrm{~m} \mathrm{~s}^{-1}$ and the minimum central sea-level pressure of $970 \mathrm{hPa}$ on 29 August when it was at approximately $1300 \mathrm{~km}$ south of Tokyo. The typhoon itself is not so severe based on size or intensity. However, record-breaking heavy rainfall brought by the typhoon caused sediment disasters, inundation, and river flooding that resulted in 82 casualties and 
16 missing people, primarily in various prefectures on Kii Peninsula such as Wakayama, Nara and Mie. The typhoon also damaged several tens of thousands of houses in Japan, also mainly on Kii Peninsula, by typhoon-triggered disasters, e.g., landslide disasters and floods (Fire and Disaster Management Agency of Japan, 2012).

The typhoon formed as a tropical depression over the sea to the west of the Mariana Islands on 23 August 2011, which moved slowly northward and reached tropical storm intensity on 25 August. On 3 September, the typhoon made landfall on Shikoku Island at 01:00 UTC. After crossing the island northward, the typhoon made landfall again in the Chugoku region of western Japan just after 09:00 UTC, 3 September. Then it moved over the Sea of Japan, accelerating its translation speed north-eastward, and then transformed into an extratropical cyclone on 5 September.

The typhoon moved so slowly that it induced continuous massive moisture advection, which brought about recordbreaking heavy rainfall over a wide area from western to northern Japan, especially along the mountains of the Kii Peninsula. At the mountainous station Kamikitayama in Nara Prefecture, an observed $1652.5 \mathrm{~mm}$ rainfall was recorded in $72 \mathrm{~h}$, which is the new high record for $72 \mathrm{~h}$ rainfall in Japan. The total rainfall amount at the station reached $1805.5 \mathrm{~mm}$, and in some areas was estimated to have a total rainfall exceeding $2000 \mathrm{~mm}$ based on radar rain gauge analyzed precipitation (Japan Meteorological Agency, 2011). The Japan Meteorological Agency's (JMA) best track data is shown in a black dashed line with squares, indicating the 6-hourly positions, in Fig. 1.

The focus of the present study is twofold. First, we determine how the potential of landslide occurrence varies if the typhoon track might perturb slightly from the best track. The amount of damage caused by a TC depends greatly on the TC track relative to the region of interest. Heavy rainfall is tied to topographic features such as mountain ranges and also depends on the typhoon track and migration speed. As such, we applied a physically consistent typhoon bogussing scheme, the potential vorticity inversion (PVI) method developed by Yoshino et al. (2008), to the typhoon, whereby a set of perturbed typhoon realizations were produced. The PVI method allows the position of the typhoon-associated potential vorticity to displace its location at a certain time in a physically consistent way. We then determine how global warming will affect typhoon intensity. Therefore, we apply the same scheme to typhoons of various intensities.

The remainder of the present study is organized as follows. In Sect. 2, we briefly describe the PVI method, the configuration of the numerical model and the design of the experiments. Sections 3 and 4 present the model simulation results, and the interpretation of these results are presented in Sect. 5. Section 6 presents our conclusions and raises several issues concerning possible future study.

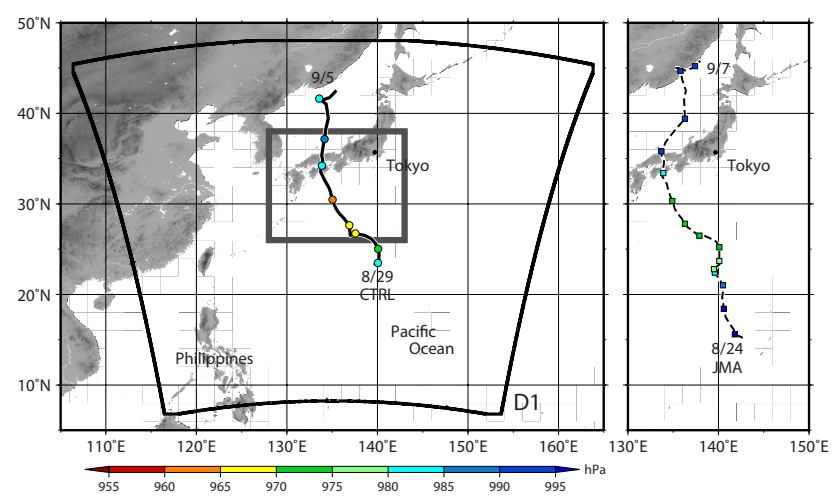

Figure 1. Computational domain 1 used in the present study except for the CTRL experiment and the tracks of Typhoon Talas (2011) from the best track data provided by JMA (black dashed line with squares) and CTRL (purple solid line with circles). Colored symbols represent the sea-level pressure of the typhoon at 00:00 UTC every day.

\section{Model configuration and simulation design}

Throughout this study a mesoscale meteorological model, i.e., the Weather Research and Forecasting (WRF) model version 3.1.1 (Skamarock et al., 2008), was used to simulate Typhoon Talas (2011). The JMA operates a real-time data assimilation system known as the JMA Climate Data Assimilation System (JCDAS). The JCDAS data set, which is one of the reanalysis data described in detail at http://jra.kishou. go.jp, has a spectral resolution of T106 (equivalent to a horizontal grid spacing of approximately $120 \mathrm{~km}$ ) and 40 vertical layers with the top at $0.4 \mathrm{hPa}$. We use the JCDAS data set as the initial and boundary conditions of the WRF model.

First, we conducted a simple hindcast simulation of the typhoon, hereinafter referred to as the CTRL simulation, driving the WRF model with 6-hourly JCDAS reanalysis data. The domain size of the CTRL simulation is $4512 \mathrm{~km} \times 4512 \mathrm{~km}$ with a $16 \mathrm{~km}$ horizontal resolution, covering a wide region from the eastern part of the Asian continent to the Japanese Islands, including the Philippine Sea (not shown, but approximately the same size as D1, as depicted in Fig. 1), with 50 vertical layers up to $5 \mathrm{hPa}$.

The WRF model includes four-dimensional data assimilation (FDDA) options using spectral nudging. This option is used in CTRL. As long as the wave numbers are limited in small values, say 1,2 or 3 , this technique ties the synoptic (large-scale) pattern to reanalysis and allows the generation of mesoscale (small-scale) disturbances without damping small-scale features. Thus, the method performs well in reducing the track error of a typhoon and enables us to hindcast a typhoon structure realistically.

The physics schemes used in the experiments are listed in Table 1. The model is initialized at 12:00 UTC, 28 August 2011 and is integrated for 8 days and 6 hours until 18:00 UTC, 5 September. The integration time steps are $60 \mathrm{~s}$. 
Table 1. WRF physics parameterization schemes used in the study.

\begin{tabular}{lll}
\hline Physics schemes & Models & References \\
\hline Microphysics & WRF single-moment six-class (WSM6) & Hong and Lim (2006) \\
Planetary boundary layer & Mellor-Yamada Level 2.5 & Janjic (2002) \\
Longwave radiation & Rapid radiative transfer model (RRTM) & Mlawer et al. (1997) \\
Shortwave radiation & Dudhia scheme & Dudhia (1989) \\
Cumulus parameterization* & Kain-Fritsch & Kain and Fritsch (1990), Kain (2004) \\
Land surface & 5-layer thermal diffusion & \\
Surface layer & Similarity theory (MM5) & \\
\hline
\end{tabular}

* Cumulus parameterization scheme was not applied to domain $3(1 \mathrm{~km})$ in NOPVI, NOPVM and $Y_{+j j} X_{+i i}$ simulations

Table 2. Specifications of simulations.

\begin{tabular}{lllll}
\hline Run name & $\begin{array}{l}\text { Boundary } \\
\text { conditions }\end{array}$ & $\begin{array}{l}\text { PVI } \\
\text { procedure }\end{array}$ & $\begin{array}{l}\text { TC } \\
\text { relocation }\end{array}$ & Description \\
\hline CTRL & JCDAS & No & - & hindcast \\
NOPVI & CTRL & No & - & WRF model evaluation \\
NOPVM & CTRL & Yes & No & PVI method evaluation \\
$\mathrm{Y}_{+j j} \mathrm{X}_{+i i}$ & CTRL & Yes & Yes & \\
\hline
\end{tabular}

The PVI method is briefly explained here. Yoshino et al. (2008) developed a method to artificially relocate the TC vortex by applying the PVI method developed by Davis and Emanuel (1991). The use of the PVI method reduces the physical imbalance of meteorological variables, as compared to other bogussing methods. A more detailed description of the PVI method used in the present study is provided in the appendix of Ishikawa et al. (2013). A new initial and boundary condition was computed for each of the typhoon relocations, and further numerical integration of WRF yielded different typhoon cases. Note that spectral nudging was not applied during the integration after the relocation so as to avoid artificial forcing on the simulated physical processes.

In order to produce an ensemble of perturbed typhoon members, the typhoon was relocated to different locations at 06:00 UTC, 1 September. At this instance, the potential vorticity was computed from the output meteorological variables of the CTRL simulation, and the TC relocation was applied. The typhoon position at the time was relocated within a range of plus or minus 30 grids, which is equivalent to $480 \mathrm{~km}$, in the east-west direction. A total of 61 basic members, including a no-perturbed case, were generated. In addition, the typhoon was relocated within a range of plus 20 grids in the east direction and minus 5 grids in the north direction (plus 5 grids in the south direction). A total of another 105 additional members were generated. Each relocation run was labeled as $Y_{+j j} X_{+i i}$, where $i i$ and $j j$ are the relocated grid distances from the original position, $Y$ indicates meridional direction and $X$ indicates zonal direction.

Using the 6-hourly output from the CTRL simulation as the initial and boundary conditions, we conduct three types of simulations as described in Table 2; one is a simple downscaling CTRL results without the PVI method, referred to hereafter as the NOPVI simulation; the second is downscaling with the PVI method but no modification on potential vorticity magnitude and position, NOPVM; and the other is downscaling with the PVI method relocating TC, $Y_{+j j} X_{+i i}$.

The performance of the modeling system is evaluated against the observations with the use of the output from NOPVI, which is a run without the use of the PVI method. The NOPVM, which is a run that uses the PVI method but not TC relocations, that is, NOPVM refers to $Y_{+00} X_{+00}$, is designed to confirm the performance and the reliability of typhoon relocation with the PVI method.

For these runs, we used three nested domains as shown in Figs. 1 and 2. The first domain covers the area of $4352 \mathrm{~km} \times 4352 \mathrm{~km}$ with a horizontal resolution of $16 \mathrm{~km}$. The second domain is $720 \mathrm{~km} \times 720 \mathrm{~km}$ with a horizontal resolution of $4 \mathrm{~km}$, and the third domain is $160 \mathrm{~km} \times 140 \mathrm{~km}$ with a resolution of $1 \mathrm{~km}$. Steep terrain affects the location and accumulation of intense rainfall (Oku et al., 2010). To reflect the effect of the steep mountains of the Kii Peninsula, the $50 \mathrm{~m}$ mesh digital elevation model (DEM) by the Geographical Survey Institute (GSI) of Japan is used to create the terrains in the finest domain. The land-use/land-category data set used is the $100 \mathrm{~m}$ mesh Geographical Information System database from the Ministry of Land, Infrastructure, Transport and Tourism (MLIT) of Japan. We do not use the Kain-Fritsch cumulus parameterization scheme in the finest domain. Other model settings are the same as those of the CTRL simulation. These runs were conducted for $96 \mathrm{~h}$ starting at 06:00 UTC, 1 September. The simulated outputs from the finest domain are stored in 5-minute intervals for the purpose of detailed analysis. The integration time steps are 60, 12 , and $3 \mathrm{~s}$ from the outermost domain to the innermost domain. 


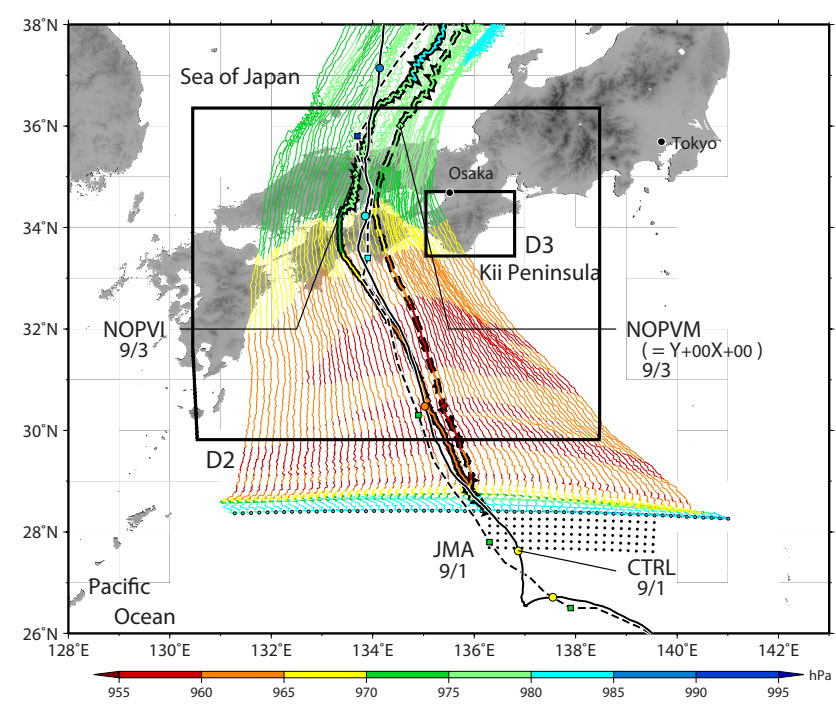

Figure 2. Computational domains 2 and 3 in the present study (except for the CTRL experiment) and the tracks of Typhoon Talas (2011) from the best track data provided by JMA (black dashed line with squares) and CTRL (black solid line with circles). Colored symbols represent the sea-level pressure of the typhoon at 00:00 UTC every day. A total of 61 solid colored lines are given by $Y_{+00} X_{+i i}$ when $-30 \leq i i \leq+30$, including NOPVM $(i i=0)$, which is indicated by the thick line bordered by the dashed black line, and NOPVI which is displayed as the thick line bordered by the solid black line. A total of 105 black dots are given by the initial location of $Y_{+j j} X_{+i i}$ when $0 \leq i i \leq+20$ and $-5 \leq j j \leq-1$.

\section{Evaluation of the WRF model performance}

\subsection{Typhoon track and intensity}

The tracks of Typhoon Talas (2011) are shown in Fig. 1 for the best track data (provided by JMA) and for the CTRL simulation, with spectral nudging imposed for the JCDAS reanalysis data. The goal of spectral nudging is to force the large-scale components of the atmospheric fields to remain close to the reanalyses, while the small-scale components are left free to evolve in the model. Since the realistic track information is embedded in the reanalysis data, the spectrally nudged track of the CTRL simulation closely follows the best track data.

NOPVI and NOPVM $\left(Y_{+00} X_{+00}\right)$ were, however, conducted without the spectral nudging option. As shown in Fig. 2, their tracks also follow the best track data closely, while NOPVM lies slightly to the east of that for the best track data. Although the reproducibility of the typhoon track route is good, the reproducibility of the translation speed is faster than reality in every case. The typhoons simulated by CTRL, NOPVI, and NOPVM move faster by factors of 1.2, 1.4 , and 1.7 compared with the observations.

In terms of the minimum of central pressure, the simulated results were deeper than the observations. The life- time minimum of simulated central pressure for the best track data was approximately $970 \mathrm{hPa}$ at 12:00 UTC, $29 \mathrm{Au}-$ gust, whereas those for CTRL, NOPVI, and NOPVM were $962 \mathrm{hPa}$ at $16: 50 \mathrm{UTC}, 961 \mathrm{hPa}$ at 19:40 UTC, and $955 \mathrm{hPa}$ at 18:55 UTC, respectively, on 1 September.

\subsection{Rainfall}

Hourly rainfall intensity and the cumulative rainfall amount from NOPVI are compared with those from Automated Meteorological Data Acquisition System (AMeDAS) observations at two record-breaking sites in the mountains (Fig. 4). At the beginning of the simulation, heavy rain area had already extended to Kamikitayama. In the simulation, heavy rain stopped approximately $12 \mathrm{~h}$ earlier than that in the observation. The duration of rainfall in the simulation is approximately 1 day shorter than that in the observation. Note that the locations of the typhoon center in the simulation translated faster than those of the best track. These differences led to the underestimation of temporal changes in the rainfall amount.

The performance of WRF in the rainfall simulation is quantitatively assessed by comparing the model spatial distribution with corresponding JMA products of Radar-AMeDAS Precipitation-gauge measurement (RAP). RAP data are raingauge calibrated radar data which have a horizontal resolution of $1 \mathrm{~km}$. Here, $r_{\mathrm{NOPVI}}$ is defined as the maximum hourly rainfall amount calculated from the output of NOPVI in each grid in the objective area, as depicted in Fig. 3. The objective area is defined so as to include major landslide sites. Similarly, $r_{\mathrm{RAP}}$ is defined as the maximum hourly rainfall amount calculated from the output of RAP. The values, $r_{\mathrm{NOPVI}}$ and $r_{\mathrm{RAP}}$, are put into the two-dimensional bins using Eq. (1). The frequency of each bin, $N(m, n)$, is counted and depicted in Fig. 5a as color tiles representing frequency, and $m$ and $n$ as increment counters.

$\left\{\begin{array}{l}(m-1) r<r_{\mathrm{RAP}} \leq m r \\ (n-1) r<r_{\mathrm{NOPVI}} \leq n r \\ r=4\left[\mathrm{mmh}^{-1}\right]\end{array}\right.$

Figure 5a compares $r_{\text {RAP }}$ and $r_{\text {NOPVI }}$ using Eq. (1) with $N(m, n)$ on the color scale, $r_{\mathrm{RAP}}$ on the $x$ axis, and $r_{\mathrm{NOPVI}}$ on the $y$ axis. If there were perfect agreement between $r_{\mathrm{RAP}}$ and $r_{\mathrm{NOPVI}}$, all points would be oriented along a straight line at a $45^{\circ}$ slope in the figure. Cases below the $45^{\circ}$ line correspond to NOPVI underestimation as well as cases above the $45^{\circ}$ line, which correspond to NOPVI overestimation. The spatial average of $r_{\mathrm{NOPVI}}$ across the objective area is $55.3 \mathrm{~mm}$, whereas that of $r_{\mathrm{RAP}}$ is $56.7 \mathrm{~mm}$. The difference in distributions between $r_{\mathrm{RAP}}$ and $r_{\mathrm{NOPVI}}$ is seen by comparing Fig. 6a and b. Extremely heavy rainfall was observed along the southeast coast of the Kii Peninsula. In the simulation, the coverage of the heavy rainfall area is limited in the mountainous region, which is brought by passage of some 


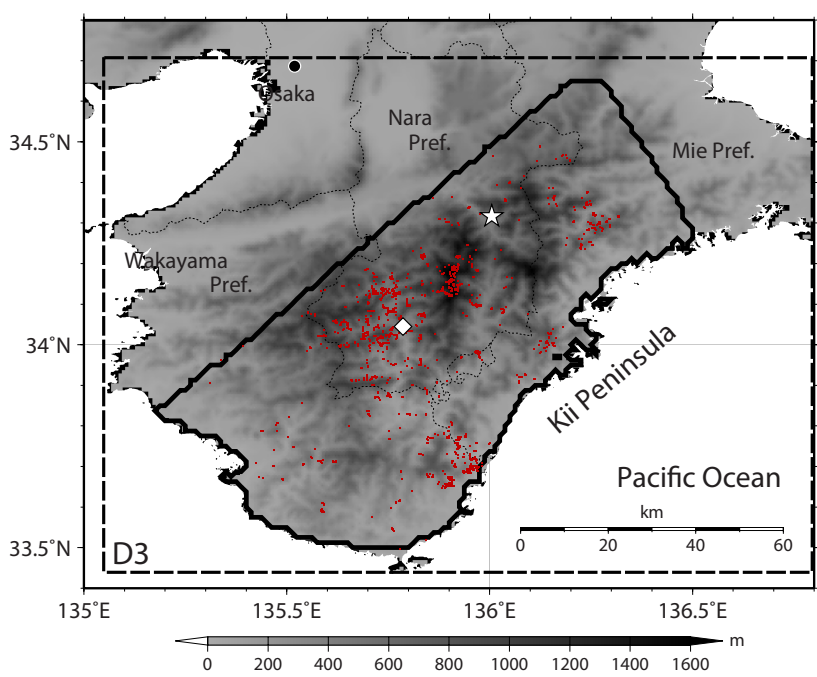

Figure 3. Location of the study area of the Kii Peninsula (solid line) and computational domain 3 of the present study (dashed line). The altitude is indicated by grayscale. The black dotted line indicates the prefectural boundary. The white star and diamond indicate the locations of Kamikitayama and Kazeya stations (AMeDAS), respectively, operated by the JMA. The red dots indicate the locations of the analyzed landslides provided by MLIT.

strong convective systems accompanying the typhoon. The difference may be partly due to the difference of low-level wind direction over the southeast slope of the Kii Peninsula. Figure $7 \mathrm{a}$ and $\mathrm{b}$ compare snapshots of low-level wind and hourly rainfall amount at the time when the typhoon landed again on western Japan. In the observed field (Fig. 7b) lowlevel southeasterly wind blows directly towards the mountain range, bringing heavy rainfall there. In the simulation, however, the wind direction was southerly so that topographic lift was rather weak. This difference partially leads to the difference rainfall pattern between Fig. $6 a$ and $b$.

Next, we evaluate the model performance for the maximum daily rainfall amount using Eq. (1) but $r=32$ $\left[\mathrm{mm} \mathrm{day}^{-1}\right.$ ] for clarity of Fig. $5 \mathrm{~b}$. A spatial average of $r_{\mathrm{NOPVI}}$ is $398.1 \mathrm{~mm}$ and that of $r_{\mathrm{RAP}}$ is $632.6 \mathrm{~mm}$. In the NOPVI simulation, in which a translation speed was about 1.4 times faster than that of the real typhoon, the spatial average of the maximum daily rainfall decreased by $63 \%$ compared to that in RAP. This indicates that the amount of rainfall has an approximately inverse relationship with the migration speed of the typhoon. Comparison of Fig. $6 \mathrm{~d}$ and e reveals that the model simulated heavy rain areas: for example, the maximum daily rainfall amounts exceeding $600 \mathrm{~mm}$, which is shown in orange color in the figure, are much narrower than those observed. This could be due to the fact that the modelsimulated typhoon moves faster than the observation.

\section{Evaluation of the PVI method performance}

Before conducting typhoon relocation experiments, the consistency of the PVI TC relocation procedure is assessed by the comparison of NOPVI and NOPVM $\left(Y_{+00} X_{+00}\right)$ cases. The vortex in NOPVM is extracted and put back at the same location again. The meteorological fields of wind velocity and potential temperature are replaced by the fields that are retrieved from PVI method. So there is a difference between NOPVM and NOPVI, which is caused by the numerical truncation error. As shown in Fig. 2, the location of the typhoon center at the initial time in the NOPVM simulation is the same as that in NOPVI, which indicates that the PVI procedure at the vortex was put back in the same place. The track of NOPVM was initially close to that of NOPVI. Several hours after the initial time, however, the track of NOPVM deviated to the east of the track of NOPVI. The minimum sea-level pressure of NOPVM became lower by several hPa compared with the NOPVI case. The PVI method acted as a low-pass filter and removed the gravity-wave in the procedures, recovering the entire meteorological field from the PV field (Yoshino et al., 2008). In this case, the PVI method is not perfectly reversible.

However, the differences in the simulated track and intensity of the typhoon between NOPVM and NOPVI fortunately seem to be acceptably small for the present purposes of finding potentially the worst-case track in respect to a heavy rainfall-induced disaster. The spatial average of the maximum hourly rainfall amount across the objective area, as shown in Fig. 3, is $56.7 \mathrm{~mm}$ for NOPVM, which is very close to the $55.3 \mathrm{~mm}$ for NOPVI. As Fig. $6 \mathrm{~b}$ and c show, there are line-shaped areas where the maximum hourly rainfall amount is greater than $70 \mathrm{~mm}$, which are brought by passage of some strong convective systems accompanying the typhoon. Comparison of Fig. $6 \mathrm{~b}$ and $\mathrm{c}$ reveals the difference between NOPVI and NOPVM in respect to the locations of heavy rain path.

This causes differences in the spatial distribution of the maximum daily rainfall amount between NOPVI and NOPVM, as depicted in Fig. 6e and f, respectively. Lineshaped areas where the maximum daily rainfall amount exceeds $500 \mathrm{~mm}$ exist in both cases, though the locations are slightly different. The spatial average of the maximum hourly rainfall across the objective area is $411.1 \mathrm{~mm}$ for NOPVM and $398.1 \mathrm{~mm}$ for NOPVI. The difference between NOPVM and NOPVI is much smaller than that of NOPVI and RAP. The simulation with the PVI method skilfully reproduces the characteristics of the rainfall simulated without the PVI method. 

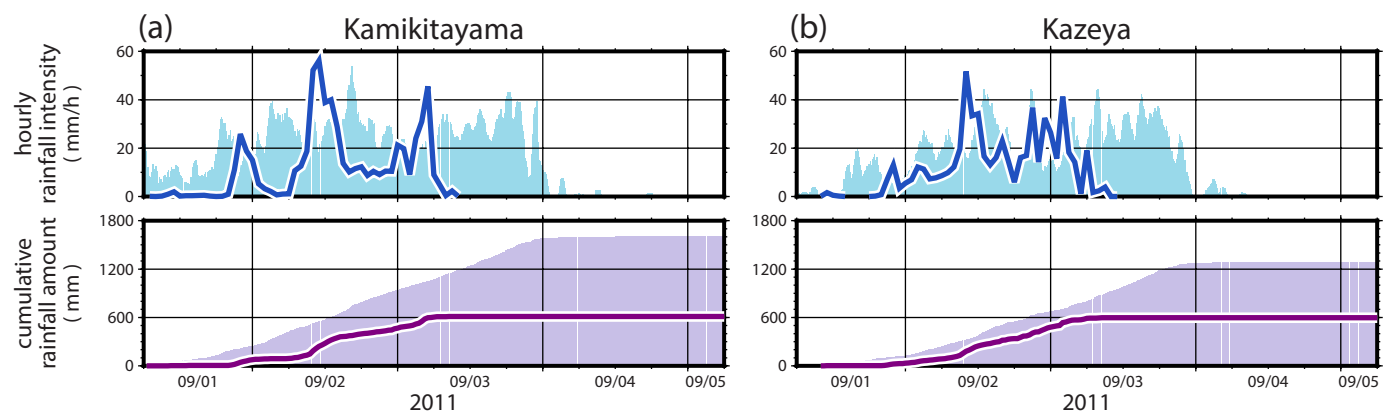

Figure 4. Temporal changes in hourly rainfall intensity (top panels) and cumulative rainfall amount from 06:00 UTC, 1 September 2011 (bottom panels) at the Kamikitayama (left panels) and Kazeya (right panels) locations for the AMeDAS observations (bars) and NOPVI results (lines). The locations of these two stations are shown in Fig. 3.
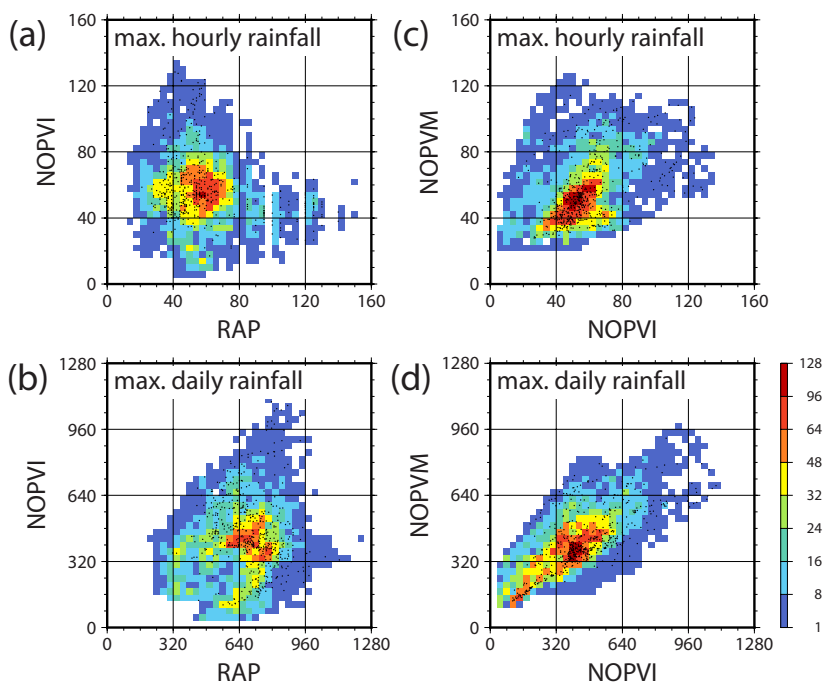

Figure 5. Scatter diagrams of NOPVI versus RAP and NOPVM versus NOPVI rainfall amounts. (a) and (c) show the maximum hourly rainfall amounts, (b) and (d) show the maximum daily rainfall amounts. The number of grid counts computed by Eq. (1) is indicated by the color scale. Dots indicate grids corresponding to landslide locations.

\section{Assessment of landslide hazard}

\subsection{Soil water index}

The soil water index (SWI; Okada et al., 2001) is an indicator of water amount in the near-surface soil layers, which is strongly related with the risk of landslide disasters. Landslide disasters frequently occur in areas of high SWI values. The risk of landslide disasters caused by heavy rainfall becomes high when the amount of moisture in soil increases. The amount of moisture in the soil is estimated using the tank model method, which indicates how much rainwater is contained in soil based on rainfall analysis. SWI is used for not only assessing the occurrence of potential landslide haz- ards (Urita et al., 2011; Saito et al., 2010), but also for early warning systems of landslide disasters (Osanai et al., 2010).

SWI is calculated using a tank model as a conceptual rainfall-runoff model. In Okada et al. (2001), SWI at a specific time $(t+\Delta t)$ equals the total storage volume $S_{k}$ of three serial tanks, in other words,

$\mathrm{SWI}(t+\Delta t)=\sum_{k} S_{k}(t+\Delta t)$,

where $k=1,2,3$, from the top tank to the bottom. Each $S_{k}$ is computed every $10 \min (\Delta t=10)$ by

$$
\begin{aligned}
& S_{k}(t+\Delta t)= \\
& \begin{cases}S_{k}(t)-\left[\sum_{l} Q_{k l}(t)+Z_{k}(t)\right]+P(\Delta t) & (k=1) \\
S_{k}(t)-\left[\sum_{l} Q_{k l}(t)+Z_{k}(t)\right]+\left[Z_{k-1}(t+\Delta t)-Z_{k-1}(t)\right] & (k \geq 2)\end{cases}
\end{aligned}
$$

where $P(\Delta t)$ is the $10 \mathrm{~min}$ rainfall amount. $Q_{k l}$ is seepage runoff volume and height of the runoff from the side $l$ th hole. The top tank has two holes on the side, others have one. $Z_{k}$ is vertical seepage volume from the bottom of the $k$ th tank. These are obtained by

$Q_{k l}(t)= \begin{cases}a_{k l}\left\{S_{k}(t)-L_{k l}\right\} & \left(S_{k}(t)>L_{k l}\right) \\ 0 & \left(S_{k}(t) \leq L_{k l}\right)\end{cases}$

$Z_{k}(t)=b_{k} S_{k}(t)$,

where $a_{k l}$ is the coefficient of runoff from the side $l$ th hole, $b_{k}$ is the coefficient of seepage from the bottom hole of the $k$ th tank, and $L_{k l}$ represents the height of the runoff on the side $l$ th hole of the $k$ th tank. Constant $a_{k l}, b_{k}$ and $L_{k l}$ used in the present study are provided in the Appendix of Okada et al. (2001).

\subsection{Track ensemble}

Severity of each ensemble member is assessed in reference to the NOPVI rainfall pattern. Three parameters are employed, 

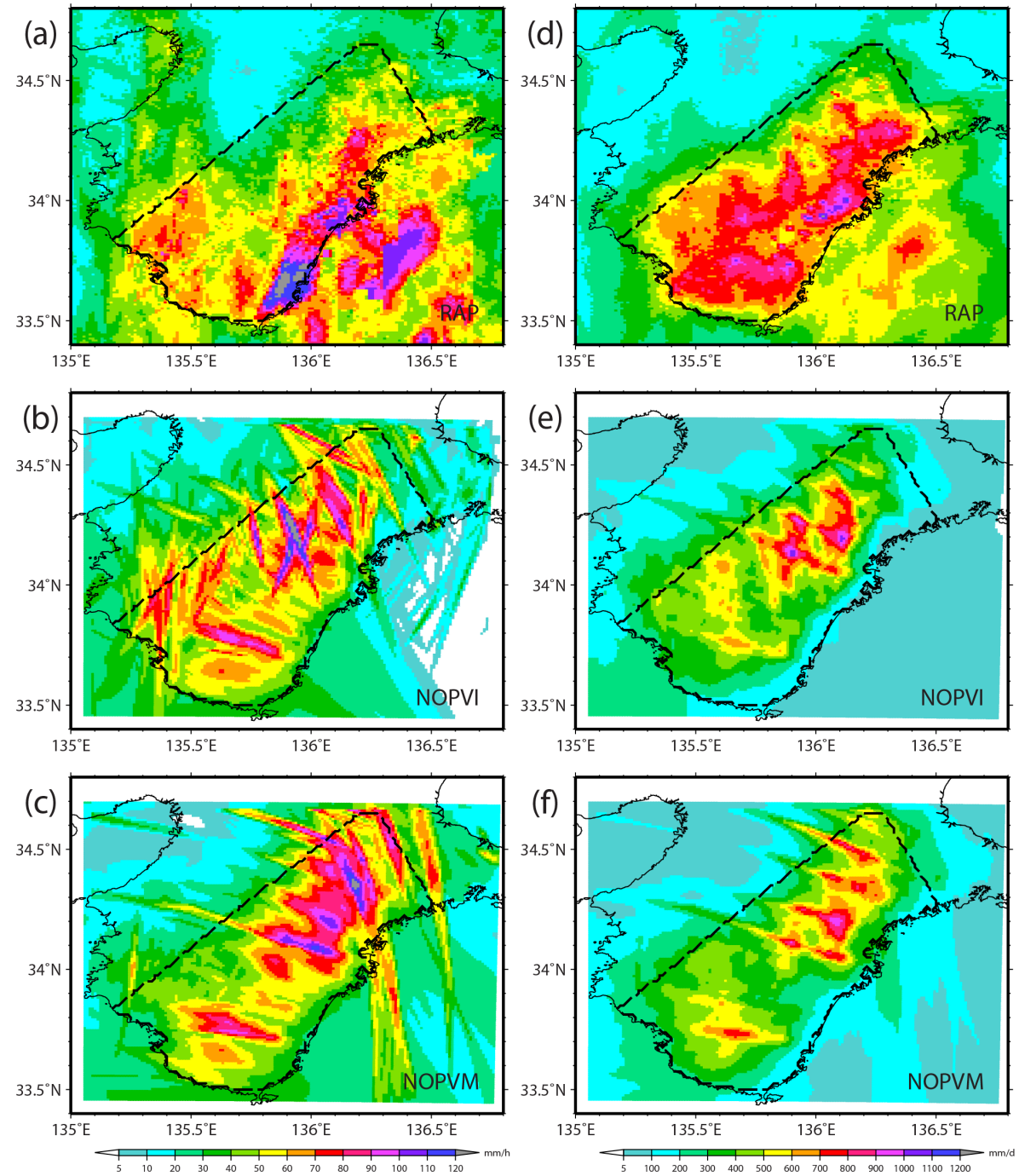

Figure 6. Horizontal distribution of RAP, NOPVI, and NOPVM rainfall amounts. (a) through (c) are the maximum hourly rainfall amounts, (d) through (f) are the maximum daily rainfall amounts. The dashed line indicates the location of the study area on the Kii Peninsula (see Fig. 3).

which are the maximum hourly rainfall amount, the maximum daily rainfall amount, and the maximum SWI. We introduce an index, $R(\alpha)$, defined by Eq. (6), where $\alpha$ is an objective quantity, such as rain intensity, accumulated rainfall and SWI.

$R(\alpha)_{Y_{+j j} X_{+i i}}=\frac{\sum \alpha_{Y_{+j j} X_{+i i}}}{\sum \alpha_{\mathrm{NOPVI}}}$

Although the model has more than a little bias as described in the previous section, the model bias for the rainfall amount with respect to the observations is expected to be reduced by using Eq. (6). An $R$ of less than 1 indicates that the rainfall impact is less than that for the NOPVI, and an $R$ of greater than 1 indicates that it is more than that for the NOPVI. Moreover, the highest $R$ is considered one of the worst-case scenarios.
In Fig. 8a, each typhoon track is colored according to $R$ for the maximum hourly rainfall amount. Figure $8 \mathrm{~b}$ and $\mathrm{c}$ show similar estimations for daily rainfall amount and SWI, respectively. Basically, the $R$ for rainfall and SWI decrease when the typhoon location is shifted to the west, i.e., with increasing distance from the target area. Reversely, they increase as the typhoon track approaches the Kii Peninsula. However, there is a critical track for achieving the maximum $R$, and $R$ decreases when the typhoon location is shifted closer to the Kii Peninsula than the critical track. It is due to the structure of the typhoon. Generally, rainbands and rings of strong convection form at a certain distance from the typhoon center. It is easy to identify the critical track of the maximum daily rainfall amount along a specific latitude from the lower panel of Fig. 8b, and $R$ is 1.18 when the typhoon is shifted to $Y_{+00} X_{+09}$. Among the 166 ensemble members, 

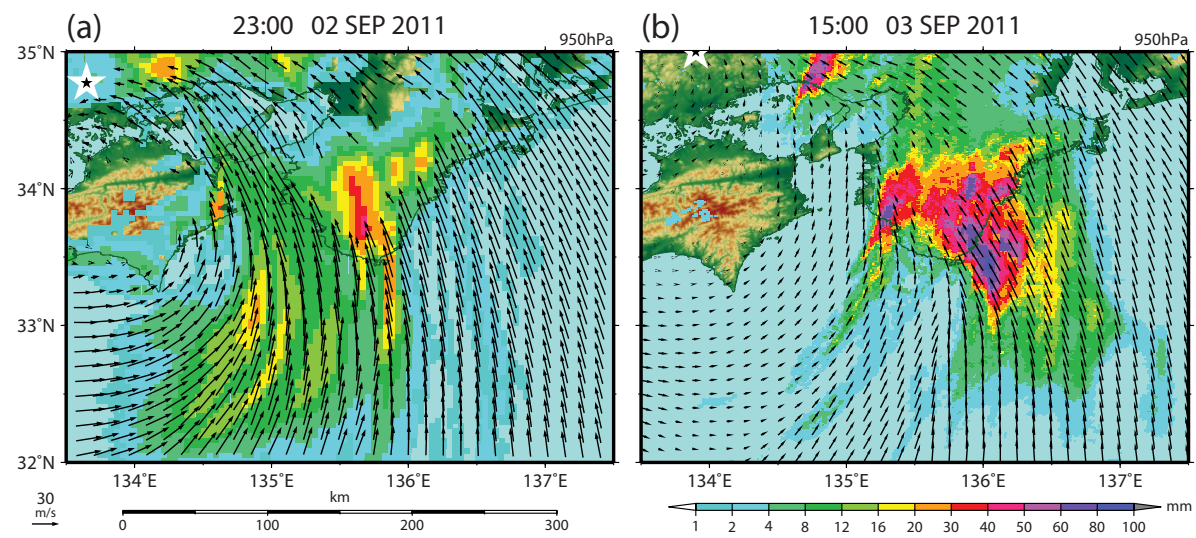

Figure 7. (a) Simulated hourly rainfall amount and wind field on $950 \mathrm{hPa}$ level at 23:00 UTC on 2 September from D2 of NOPVI, (b) observed RAP and analyzed wind field produced by the JMA/MSM (Meso Scale Model) at 15:00 UTC. These are when the typhoon made landfall again at the Chugoku region of western Japan, the location is depicted by the star symbol.

(a)

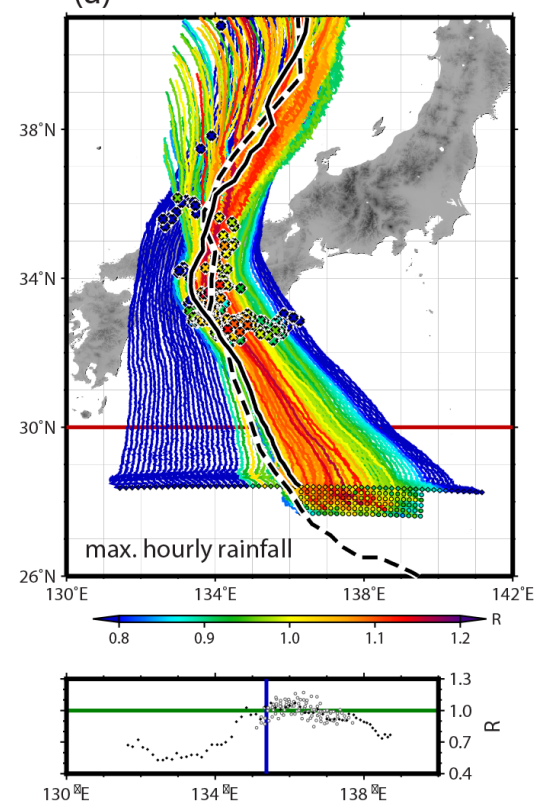

(b)

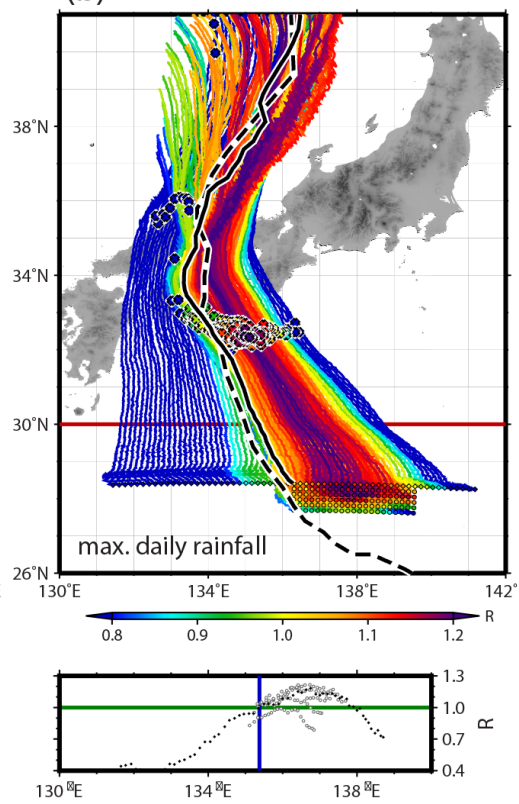

(c)
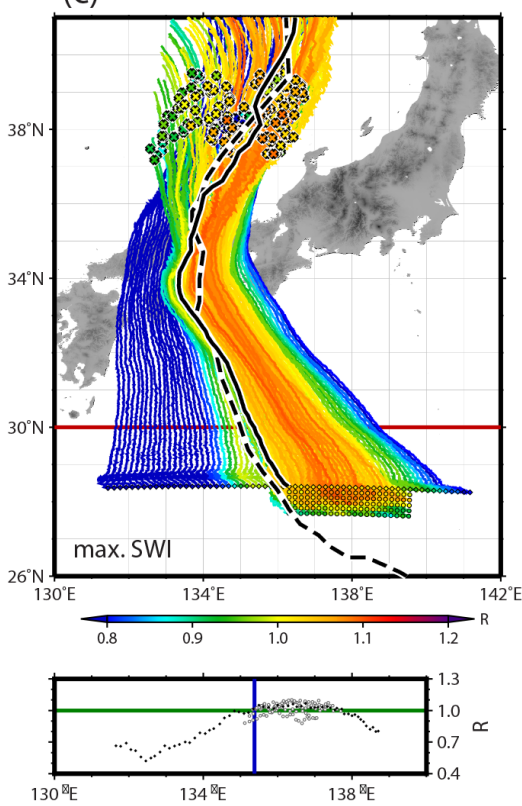

Figure 8. Severity of each ensemble case estimated for different measures: (a) maximum hourly rainfall amount, (b) maximum daily rainfall amount, and (c) maximum soil water index (SWI). The color of the track for each case represents the $R$ score defined by Eq. (6). The crosses indicate the TC location when maximum rainfall or SWI occurs. The filled diamonds (open circles) in the lower panels indicate the $R$ score when each typhoon in a suit of 61 basic members ( 105 additional members) passed $30^{\circ} \mathrm{N}$, as indicated by the red line in the upper panels. In the lower panels, the green line corresponds to $R=1$. The vertical blue line corresponds to the longitude at which the typhoon simulated by NOPVI passed at $30^{\circ} \mathrm{N}$.

the maximum value of $R$ is 1.21 when the typhoon is shifted to $Y_{-02} X_{+08}$. For the maximum hourly rainfall amount in Fig. 8a, in contrast, $R$ is not so peaked but it extends from 135 to $137^{\circ} \mathrm{E}$ when the typhoon was at $30^{\circ} \mathrm{N}$.

One of the strongest convective systems existed there, which brought heavy rainfall in the northeast quadrant of the Typhoon Talas (2011). This strong convective system came across the southeastern slope directly, so that a high
$R$ value was computed in reality. In NOPVI, however, the major rainfall area came across the southwestern slopes, so that eastward-shifted ensembles had $R$ values higher than NOPVI. Shifting the typhoon by $160 \mathrm{~km}$ to the east at the relocation time increases the possibility that an $R$ peak will occur, although there is no recognizable relationship between the maximum $R$ and the typhoon location. In this case, the maximum $R$ of the maximum hourly rainfall amount 

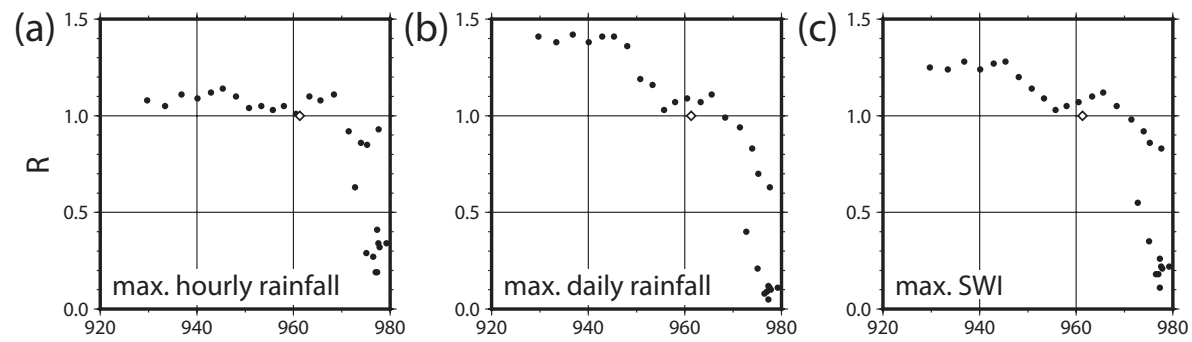

Figure 9. Relationship between the minimum central sea-level pressure of the typhoon ( $x$ axis [units: hPa]) and $R$ ( $y$ axis) for (a) the maximum hourly rainfall amount, (b) the maximum daily rainfall amount, and (c) the maximum SWI.

occurred when the typhoon was shifted to $\mathrm{Y}_{-03} \mathrm{X}_{+10}$ with a value of 1.17 . The appearance of a heavy rain area accompanied by convective clouds in the outer rainband is certainly unsystematic and sporadic. The intensity of the individual convective clouds was sufficient to have caused localized rainfall. The $R$ of the maximum SWI, as depicted in Fig. 8c, shows the superimposed characteristics of the $R$ of the hourly rainfall amount and the $R$ of the daily rainfall amount. A peak occurred when the typhoon was shifted at $Y_{-03} X_{+10}$ with a value of 1.10 . This implies that there is a $10 \%$ increase in the risk of a landslide disaster occurring, depending on the typhoon track.

\subsection{Intensity ensemble}

A number of researchers have reported that future TCs are likely to become more intense, with larger peak wind speeds and heavier precipitation associated with ongoing sea surface temperature increases (e.g., IPCC, 2007; Oouchi et al., 2006). It is important to assess how intensity changes in TCs can influence the hazard of heavy rainfall-induced disasters. In the present study, we focus on Typhoon Talas (2011) and modify the potential vorticity intensity of the typhoon using the PVI method without changing the location. For this purpose, a series of typhoons of different intensities perturbing from the original are generated. This is done by changing the potential vorticity intensity when putting the typhoon potential vorticity back to its original location in the NOPVM experiment. Note that the modification of typhoon intensity did not influence its track route much, although this is not shown herein.

In changing the intensity of the potential vorticity of the simulated typhoons, we do not change the mixing ratio of water vapor within the specified TC potential vorticity, nor in the surrounding field. This is based on the idea that precipitable water content should remain unchanged in order to eliminate possible influences of precipitable water change on rainfall amount. Under this assumption, relative humidity within the TC potential vorticity decreases when the potential vorticity is intensified, because the temperature within the increased TC potential vorticity becomes higher. It is noted that another idea would be to set the relative humidity within TC to unchanged; in this case, however, precipitable water content is increased, which itself may affect the rainfall amount. In the present study, we focus on the effects of the increase of TC intensity but not on the effects of the increase of precipitable water content.

Using the output from a set of typhoons of different intensity, $R$ of Eq. (6) is plotted in Fig. 9 as a function of the minimum central sea-level pressure achieved during the simulation. Some cases, in which typhoons did not develop from the initial state, are excluded. For these members the modification (intensification) to the vortex might be unrealistically strong to allow the model physics to sustain the intensity, so that the vortex could not develop further. Thus, we exclude these ensemble members from the assessment.

As the typhoon intensity decreases, i.e., as the minimum central sea-level pressure of the typhoon increases, $R$ is expected to decrease. The $R$ of the maximum hourly rainfall amount reaches a blunt peak of 1.14 at $945 \mathrm{hPa}$ of the minimum central sea-level pressure of the typhoon. Figure 9a shows that $R$ does not strongly depend on the typhoon intensity in the range of less than $960 \mathrm{hPa}$. However, the maximum daily rainfall amount is dependent on the intensity of the typhoon. Figure $9 \mathrm{~b}$ indicates that $R$ increases with decreasing minimum central sea-level pressure of the typhoon and reaches a peak of 1.42 at $936 \mathrm{hPa}$. However, below $936 \mathrm{hPa}$, $R$ increases very little.

The higher potential vorticity causes the warmer vortex core of TC, hence TC can hold much more water vapor from the sea surface. Consequently, it brings a more accumulated rainfall amount, but is not considered to always lead severer rainfall intensity. Figure 9 implies there is the limitation of convective cloud growth and it might suppress further intensification of rainfall. As in the case of the track ensemble, $R$ of the maximum SWI, as depicted in Fig. 9c, shows the superimposed characteristics of the hourly rainfall amount and the daily rainfall amount. The maximum value of $R$ at $936 \mathrm{hPa}$ is 1.28 . 


\section{Conclusions}

In the present paper, we proposed an approach to assess the potential for heavy rainfall induced by TCs with controlled track and intensity in order to find out a worst-case scenario for disaster hazards. This approach involves the assessment of the occurrence hazard of heavy rainfall-induced disasters, for instance landslide disasters. We apply the approach to Typhoon Talas (2011) as a case study. Using the PVI method, an ensemble of 166 perturbed typhoons was produced using a mesoscale meteorological model. The SWI for the occurrence hazard of a landslide disaster was calculated for each of the perturbed cases. In the severest cases, the spatially averaged SWI was 1.10 times as large as that of the original typhoon. In order to demonstrate quantitatively the potential damage due to a landslide for typhoons of various intensities, a procedure was proposed to generate different typhoon intensities that perturb. The maximum spatially averaged SWI is 1.28 times as large as that of the original typhoon intensity.

Thus, 1.10 times 1.28 approximately equals 1.4 , which is a simplistic answer to the maximum $R$ if both track and intensity of the typhoon are changed. However, an ensemble simulation that simultaneously considers variations in both the track and the potential vorticity intensity of the typhoon should be conducted. In addition, the migration speed of the typhoon is an important factor that affects the rainfall amount. Yen et al. (2011) performed a simulation in which the migration speed of Typhoon Morakot (2009) was doubled and found that the maximum accumulated rainfall over Taiwan decreased by $33 \%$ as compared to the control simulation. In this present case we tried several combinations of physical parameterization, but we could not reproduce the slow migration unless spectral nudging was applied. The more fundamental study to reveal the cause of the slow movement is required, in addition to the present application study.

The scenario-based extreme event estimation conducted herein provides no information on the probability of occurrence or the return period. The extreme estimation conducted herein is not directly intended for preventive or mitigative planning, which require knowledge of the probability of occurrence or the return period. However, it is hoped that the estimation of the present study will contribute to understanding of extreme events by providing a set of scientifically possible examples based actual TCs. It is hard to specify the worst-case possible scenario which involves TCs in the future, since we can assume a tremendous number of tracks and intensities of TC. Consideration based on a past TC disaster as presented in this study helps not only policymakers but also those who are not familiar with a TC to imagine the worst-case possible scenario.
Acknowledgements. The present study was conducted under the framework of the 'Precise Impact Assessments on Climate Change' of the Program for Risk Information on Climate Change (SOUSEI Program) supported by the Ministry of Education, Culture, Sports, Science, and Technology (MEXT) of Japan and by a Scientific Research Grant (No. 23246090) from the Japan Society for Promotion of Sciences (JSPS). The simulations of the present study were performed on the supercomputer of the Academic Center for Computing and Media Studies (ACCMS), Kyoto University.

Edited by: K. Chang

Reviewed by: two anonymous referees

\section{References}

Davis, C. A. and Emanuel, K. A.: Potential Vorticity Diagnostics of Cyclogenesis, Mon. Weather Rev., 119, 1929-1953, 1991.

Dudhia, J.: Numerical study of convection observed during the winter monsoon experiment using a mesoscale two-dimensional model, J. Atmos. Sci., 46, 3077-3107, 1989.

Fire and Disaster Management Agency of Japan: Damages Caused by Typhoon No. 12, 2011, 20th Report on 28 September 2012, http://www.fdma.go.jp/bn/2012/detail/731.html (last access: 26 Septemer 2014), 2012.

Hong, S.-Y. and Lim, J.-O. J.: The WRF single-moment 6-class microphysics scheme (WSM6), J. Korean Meteorol. Soc., 42, 129151, 2006.

IPCC: Climate Change 2007: The Physical Science Basis, Contribution of Working Group I to the Fourth Assessment Report of the Intergovernmental Panel on Climate Change, Cambridge University Press, Cambridge, UK and New York, NY, USA, 2007.

IPCC: Managing the Risks of Extreme Events and Disasters to Advance Climate Change Adaptation, A Special Report of Working Groups I and II of the Intergovernmental Panel on Climate Change, Cambridge University Press, Cambridge, UK and New York, NY, USA, 2012.

Ishikawa, H., Oku, Y., Kim, S., Takemi, T., and Yoshino, J.: Estimation of a possible maximum flood event in the Tone River basin, Japan caused by a tropical cyclone, Hydrol. Process., 27, 3292-3300, 2013.

Janjic, Z. I.: Nonsingular implementation of the Mellor-Yamada Level 2.5 scheme in the NCEP Meso model, NCEP Office Note Tech. Rep. 437, Camp Springs, MD, USA, 2002.

Japan Meteorological Agency: Annual Report on the Activities of the RSMC Tokyo - Typhoon Center 2011, http://www.jma.go.jp/jma/jma-eng/jma-center/rsmc-hp-pub-eg/ AnnualReport/2011/Text/Text2011.pdf (last access: 26 Septemer 2014), 2011.

Kain, J. S.: The Kain-Fritsch convective parameterization: An update, J. Appl. Meteorol., 43, 170-181, 2004.

Kain, J. S. and Fritsch, J. M.: A one-dimensional entraining/detraining plume model and its application in convective parameterization, J. Atmos. Sci., 47, 2784-2802, 1990.

Mlawer, E. J., Taubman, S. J., Brown, P. D., Iacono, M. J., and Clough, S. A.: Radiative transfer for inhomogeneous atmospheres: RRTM, a validated correlated-k model for the longwave, J. Geophys. Res., 102, 16663-16682, 1997. 
Okada, K., Makihara, Y., Shimpo, A., Nagata, K., Kunitsugu, M., and Saito, K.: Soil Water Index, Tenki, 47, 36-41, 2001.

Oku, Y., Takemi, T., Ishikawa, H., Kanada, S., and Nakano, M.: Representation of Extreme Weather during a Typhoon Landfall in Regional Meteorological Simulations: A Model Intercomparison Study for Typhoon Songda (2004), Hydrol. Res. Lett., 4, 1-5, doi:10.3178/hrl.4.1, 2010.

Oouchi, K., Yoshimura, J., Yoshimura, H., Mizuta, R., Kusunoki, S., and Noda, A.: Tropical Cyclone Climatology in a GlobalWarming Climate as Simulated in a $20 \mathrm{~km}-$ Mesh Global Atmospheric Model: Frequency and Wind Intensity Analyses, J. Meteorol. Soc. Jpn., 84, 259-276, doi:10.2151/jmsj.84.259, 2006.

Osanai, N., Shimizu, T., Kuramoto, K., Kojima, S., and Noro, T.: Japanese early-warning for debris flows and slope failures using rainfall indices with Radial Basis Function Network, Landslides, 7, 325-338, doi:10.1007/s10346-010-0229-5, 2010.

Saito, H., Nakayama, D., and Matsuyama, H.: Two Types of Rainfall Conditions Associated with Shallow Landslide Initiation in Japan as Revealed by Normalized Soil Water Index, Sci. Online Lett. Atmos., 6, 57-60, doi:10.2151/sola.2010-015, 2010.
Skamarock, W. C., Klemp, J. B., Dudhia, J., Gill, D. O., Barker, D. M., Duda, M. G., Huang, X.-Y., Wang, W., and Powers, J. G.: A Description of the Advanced Research WRF Version 3, Tech. Rep. NCAR/TN-475+STR, NCAR Tech. Note, Boulder, CO, USA, 2008.

Urita, S., Saito, H., Nakayama, D., Izumi, T., and Matsuyama, H.: Spatial Distribution and Regional Characteristics of Heavy Rainfall That Enhanced Potential Landslide Hazard Occurrence in Japan as Revealed by Soil Water Index, J. Geogr. (Chigaku Zasshi), 120, 615-630, doi:10.5026/jgeography.120.615, 2011.

Yen, T.-H., Wu, C.-C., and Lien, G.-Y.: Rainfall simulations of Typhoon Morakot with controlled translation speed based on EnKF data assimilation, J. Terr.-Atmos. Ocean. Sci., 22, 647660, 2011.

Yoshino, J., Kojima, H., and Yasuda, T.: Development of a new typhoon bogussing scheme based on potential vorticity dynamics for highly accurate typhoon forecasts, Kaigan-KogakuRonbunsyu, 55, 436-440, 2008. 